\title{
Resiliência e Mecanismos de Defesa em Pacientes com Câncer em Quimioterapia Ambulatorial
}

https://doi.org/10.32635/2176-9745.RBC.2022v68n1.1557

Resilience and Defense Mechanisms in Patients with Cancer Outpatient Chemotherapy
Resiliencia y Mecanismos de Defensa en Pacientes con Cáncer en Quimioterapia Ambulatorial

Júlia Mariá Azambuja Santos'; Ana Maria Vieira Lorenzzoni²; Aline Tigre ${ }^{3}$; Elizeth Heldt ${ }^{4}$

RESUMO

Introduçáo: A capacidade de enfrentamento de situaçôes adversas, que é definida como resiliência, auxilia o paciente a superar as dificuldades do tratamento. Entretanto, ainda são poucos os estudos que avaliam a resiliência em pacientes com câncer que realizam quimioterapia ambulatorial. Objetivo: Avaliar a resiliência de pacientes com câncer em tratamento quimioterápico ambulatorial e verificar a correlação com os mecanismos de defesa, sintomas depressivos e de ansiedade. Método: Estudo observacional, de correlação e prospectivo, com pacientes com diagnóstico de câncer, de ambos os sexos, maiores de 18 anos, alfabetizados e em início de tratamento com quimioterapia ambulatorial. Os seguintes instrumentos foram aplicados no primeiro dia de tratamento quimioterápico e após 30 a 45 dias: Escala de Resiliência, Defense Style Questionnaire (DSQ-40), Inventário de Depressão de Beck e Inventário de Ansiedade de Beck. Resultados: Um total de 55 participantes foi incluído, sendo 32 (58\%) do sexo feminino, com média e desvio-padrão (DP) de idade de 54,1 (DP=12,2) anos. Os diagnósticos mais frequentes foram câncer colorretal, 15 (27\%) e câncer de mama, 12 (22\%). Observou-se correlação negativa significativa entre sintomas depressivos e de ansiedade com os níveis de resiliência tanto na primeira ( $<<0,001)$ como na segunda avaliação $(\mathrm{p}<0,05)$. Os mecanismos de defesa maduros (humor e racionalização) apresentaram correlação positiva significativa e os imaturos (atuação e cisão) demonstraram correlação negativa. Conclusão: Os resultados confirmaram que a maior capacidade de resiliência se correlaciona com o uso de mecanismos de defesa adaptativos e com menores níveis de sintomas depressivos e de ansiedade em pacientes durante a quimioterapia ambulatorial.

Palavras-chave: resiliência psicológica; neoplasias; tratamento farmacológico; mecanismos de defesa.

\section{ABSTRACT}

Introduction: The ability to cope with adverse situations, defined as resilience, helps patients to overcome difficulties in their treatment. However, there are still few studies assessing resilience in cancer patients undergoing outpatient chemotherapy. Objective: To assess resilience in cancer patients undergoing outpatient chemotherapy and to verify its correlation with defense mechanisms, as well as with symptoms of depression and anxiety. Method: Observational, correlational and prospective study with patients of both sexes, diagnosed with cancer, over 18 years old, literate and beginning treatment with outpatient chemotherapy. The following instruments were applied on the first day of chemotherapy treatment and then again after 30 to 45 days: Resilience Scale, Defense Style Questionnaire (DSQ-40), Beck's Depression Inventory and Beck's Anxiety Inventory. Results: A total of 55 participants were included in the study, 32 (58\%) of which were female, with an average age and standard deviation (SD) of $54.1(\mathrm{SD}=12.2)$ years. The most frequent diagnoses were colorectal cancer, $15(27 \%)$ and breast cancer, $12(22 \%)$. There was a significant negative correlation between symptoms of depression or anxiety and resilience levels both in the first $(\mathrm{p}<0.001)$ and in the second evaluation $(\mathrm{p}<0.05)$. Mature defense mechanisms (mood and rationalization) showed a significant positive correlation with resilience, while immature ones (performance and split) showed a negative correlation. Conclusion: The results confirmed that a greater capacity for resilience correlates with the use of adaptive defense mechanisms and with lower levels of depressive symptoms and anxiety in patients while receiving outpatient chemotherapy.

Key words: resilience, psychological; neoplasms; drug therapy; defense mechanisms.

\section{RESUMEN}

Introducción: La capacidad de afrontar situaciones adversas, que se define como resiliencia, ayuda al paciente a superar las dificultades del tratamiento. Sin embargo, todavía hay pocos estudios que evalúen la resiliencia en pacientes con cáncer sometidos a quimioterapia ambulatoria. Objetivo: Evaluar la resiliencia de los pacientes oncológicos sometidos a quimioterapia ambulatoria y verificar la correlación con los mecanismos de defensa y con síntomas depresivos o de ansiedad. Método: Estudio observacional, correlacional y prospectivo con pacientes de ambos sexos, diagnosticados con cáncer, mayores de 18 ańos, alfabetizados e iniciando tratamiento con quimioterapia ambulatoria. Los siguientes instrumentos se aplicaron el primer día de tratamiento de quimioterapia y otra vez después de 30 a 45 días: Escala de resiliencia, Cuestionario de estilo de defensa (DSQ-40), Inventario de depresión de Beck e Inventario de ansiedad de Beck. Resultados: Se incluyeron 55 participantes en la investigación, 32 (58\%) de los cuales eran mujeres, con una edad promedio y desviación estándar (DE) de 54,1 $(\mathrm{DE}=12,2)$ años. Los diagnósticos más frecuentes fueron cáncer colorrectal, 15 (27\%) y cáncer de mama, 12 (22\%). Se encontró una correlación negativa significativa entre síntomas depresivos o de ansiedad y los niveles de resiliencia tanto en la primera $(\mathrm{p}<0,001)$ como en la segunda evaluación $(\mathrm{p}<0,05)$. Los mecanismos de defensa maduros (estado de ánimo y racionalización) mostraron una correlación positiva significativa y los inmaduros (rendimiento y división) mostraron una correlación negativa. Conclusión: Los resultados confirmaron que una mayor capacidad de resiliencia se correlaciona con el uso de mecanismos de defensa adaptativos y con menores niveles de síntomas depresivos y ansiedad en pacientes durante la quimioterapia ambulatoria.

Palabras clave: resiliencia psicológica; neoplasias; quimioterapia; mecanismos de defensa.

1'Irmandade Santa Casa de Misericórdia de Porto Alegre, Unidade de Quimioterapia. Porto Alegre (RS), Brasil. E-mail: julia.azambuja15@gmail.com. Orcid iD: https:// orcid.org/0000-0001-7777-714X

${ }^{2,3}$ Hospital de Clínicas de Porto Alegre, Unidade de Quimioterapia. Porto Alegre (RS), Brasil. E-mails: alorenzzoni@hcpa.edu.br; atigre@hcpa.edu.br. Orcid iD: https://orcid.org/0000-0003-3989-9124; Orcid iD: https://orcid.org/0000-0003-3175-4465

${ }^{4}$ Universidade Federal do Rio Grande do Sul (UFRGS), Escola de Enfermagem. Porto Alegre (RS), Brasil. E-mail: eheldt@hcpa.edu.br. Orcid iD: https://orcid.org/00000002-4687-282X

Endereço para correspondência: Júlia Mariá Azambuja Santos. Rua Tibúrcio de Azevedo, 731/301 - Americana. Porto Alegre (RS), Brasil. CEP 94820-480. E-mail: julia.azambuja15@gmail.com 


\section{INTRODUÇÃO}

O câncer está entre as doenças não transmissíveis que atualmente impactam na mudança do perfil de adoecimento da populaçáo brasileira e é considerado um problema de saúde pública ${ }^{1}$. Uma das modalidades de tratamentos para o câncer é a quimioterapia, que se caracteriza por ser sistêmica, composta por agentes químicos, isolados ou combinados, administrados em intervalos, que variam de acordo com os protocolos terapêuticos ${ }^{1,2}$. Ao mesmo tempo em que viabiliza a cura de alguns tumores, a quimioterapia provoca inúmeros efeitos colaterais. $\mathrm{O}$ tratamento, ainda, acarreta visitas frequentes ao hospital, levando o indivíduo a uma ruptura com o ambiente habitual, modificando costumes, capacidade de autorrealização, cuidado pessoal e interrupçôes das atividades cotidianas desempenhadas pelos pacientes ${ }^{3}$.

Como consequência da condição do diagnóstico, da associação com a morte e dos efeitos colaterais do tratamento, o paciente oncológico pode apresentar sintomas de ansiedade e depressão ${ }^{4}$. Dessa forma, desafia os profissionais da saúde a compreenderem e a identificarem os sentimentos do paciente frente ao complexo processo saúde-doença.

Define-se ansiedade como sendo uma resposta emocional a um estressor, cuja fonte náo é conhecida. É uma pressão externa colocada sobre o indivíduo, podendo ser patológica, quando interferir na eficácia da vida ou no conforto emocional razoável do indivíduo ${ }^{4}$. O paciente com sintomas depressivos apresenta perda de prazer, associados com alteraçáo do sono, do peso e do comportamento alimentar, fadiga, prejuízo cognitivo, sentimento de desvalia, de culpa e ideação suicida ${ }^{5}$.

De fato, a descoberta do diagnóstico do câncer altera a vida do paciente, gerando conflitos e incertezas. Também, a condição de portador de câncer exige que se encontrem estratégias para enfrentar as adversidades físicas e psicossociais relacionadas à patologia e ao tratamento ${ }^{6}$.

A resiliência é a capacidade individual de enfrentamento de situaçóes adversas e de problemas. Em um estudo com pacientes diagnosticados com câncer, foi verificado que a resiliência elevada promove uma adaptaçáo e flexibilidade em relação ao processo de adoecimento com melhor adesão ao tratamento ${ }^{7}$.

Os mecanismos de defesa, que são processos mentais, também, podem ser utilizados no processo de enfrentamento do câncer. Empregados pelo ego perante ameaça à integridade biológica ou psicológica, os mecanismos defensivos contribuem para o ajustamento, a adaptação e o equilíbrio da personalidade ${ }^{8}$. Ou seja, todo indivíduo seleciona inconscientemente um número restrito de comportamentos defensivos, que utiliza para lidar com os conflitos do mundo interno ou externo. Assim, os mecanismos de defesa desempenham uma função protetora e adaptativa?.

Os mecanismos de defesa maduros são considerados adaptativos, uma vez que conseguem maximizar a gratificação e possibilitam o reconhecimento consciente dos sentimentos, das ideias e suas consequências. $\mathrm{O}$ uso desses mecanismos indica uma adaptação mais favorável no manejo de eventos estressores. Nos mecanismos neuróticos, ocorre a manutenção de ideias, sentimentos, desejos, lembranças que são consideradas ameaçadoras e fora da consciência. As defesas imaturas são consideradas narcisistas e envolvem distorçôes da autoimagem e da imagem dos outros, mantendo os estressores, impulsos, ideias e afetos fora da consciência ${ }^{8,9}$.

Portanto, este estudo justifica-se em razão da necessidade de aprimorar o conhecimento das reaçóes emocionais e condiçôes de enfrentamento de pacientes com câncer em tratamento quimioterápico para qualificar o cuidado, e tem por objetivo avaliar a resiliência de pacientes com câncer em tratamento quimioterápico ambulatorial e verificar a correlação entre o nível de resiliência com os mecanismos de defesa e sintomas depressivos e de ansiedade.

\section{MÉTODO}

Estudo observacional, de correlação e prospectivo, realizado no ambulatório de quimioterapia do Hospital de Clínicas de Porto Alegre (HCPA), entre maio e agosto de 2018. Foram incluídos no estudo pacientes de ambos os sexos, maiores de 18 anos, alfabetizados e em início de tratamento com quimioterapia ambulatorial. Como critérios de exclusão, não foram elegíveis pacientes com diagnóstico de transtorno mental ou com déficit cognitivo. O convite para participar da pesquisa ocorreu antes de iniciar a primeira infusáo de quimioterapia ambulatorial, durante a consulta de enfermagem ${ }^{2}$ realizada em local reservado. $\mathrm{O}$ estudo foi aprovado pelo Comitê de Ética em Pesquisa (CEP) do HCPA sob o CAAE: 84903718.8.0000.5327 e os participantes assinaram o Termo de Consentimento Livre e Esclarecido (TCLE).

Para uniformizar a coleta de dados, foi elaborado um protocolo com informaçôes sociodemográficas (sexo, idade, nível educacional em anos de estudo) que o próprio paciente respondeu. As informaçóes clínicas (diagnóstico e tratamento quimioterápico) foram coletadas do prontuário on-line. Os seguintes instrumentos foram aplicados no primeiro dia de tratamento quimioterápico e após 30 a 45 dias: Inventário de Depressão de Beck 
(BDI), Inventário de Ansiedade de Beck (BAI), Escala de Resiliência, e o Defense Style Questionnaire (DSQ-40).

Para identificar a presença de sintomas depressivos e de ansiedade, foram utilizados o BDI e o BAI, respectivamente. Os instrumentos sáo de autorrelato, validados para o português ${ }^{10}$. Cada instrumento possui 21 itens, incluindo sintomas e atitudes que ocorreram na última semana. A intensidade dos sintomas avaliada em cada item varia de 0 a 3 , sendo que, quanto maior os escores totais do BDI e do BAI, mais intenso são os sintomas.

A Escala de Resiliência é um instrumento de autorrelato, validado para o português, e usado para medir os níveis de adaptação psicossocial frente a eventos de vida ${ }^{11}$. É composta por 25 itens descritos de forma positiva com resposta tipo Likert, variando de 1 (discordo totalmente) a 7 (concordo totalmente). Os escores da escala oscilam de 25 a 175 pontos e os valores altos indicam elevada capacidade de resiliência.

O DSQ-40 é um instrumento de autorrelato, validado para o português?. Avalia 20 mecanismos de defesa divididos em três fatores: maduros, neuróticos e imaturos. Os escores de cada mecanismo são calculados pela média de dois itens disponíveis para cada defesa e os fatores são calculados pela média dos escores das defesas que pertencem a cada fator. Cada item é avaliado por uma escala Likert que varia de 1 (discorda completamente) a 9 (concorda completamente). Considera-se que, quanto maior o escore, maior o uso do mecanismo de defesa.

O tamanho amostral foi calculado no WinPepi v.11.44. Considerando um nível de significância de 5\%, com um poder de $90 \%$, um desvio-padrão (DP) antes e depois de 2,8 e com uma correlação de 0,7 . A amostra calculada foi de 55 participantes, considerando uma diferença de 6 pontos na Escala de Resiliência.

Os dados foram analisados pelo programa estatístico Statistical Package for the Social Sciences (SPSS), versão 18.0, com nível de significância adotado de $\alpha=0,05$ e estáo apresentados como média e DP para variáveis contínuas com distribuição normal e mediana (intervalo interquartil) para variáveis assimétricas. As variáveis categóricas foram apresentadas como frequência absoluta e percentual. As mudanças na intensidade dos sintomas, ao longo da quimioterapia, foram avaliadas com o teste t ou Wilcoxon.

As correlaçôes entre a resiliência e as demais variáveis foram verificadas com o teste de correlação de Spearman e consideraram-se achados relevantes os coeficientes significativos acima de $r>0,300$. Os intervalos de tamanho de efeito de correlação do presente estudo foram: coeficiente $r=0,100$ a $<0,300$ : fraca; $r \geq 0,300$ a $<0,500$ : moderada; $r \geq 0,500$ a 1,000 : grande $^{12}$.

\section{RESULTADOS}

Um total de 55 participantes foi incluído e as características da amostra estáo apresentadas na Tabela 1. Houve uma perda na segunda avaliação em virtude da má adesão do paciente ao tratamento. Observou-se que 32 (58\%) eram do sexo feminino, com média e DP de idade de 54,1 (DP=12,2) anos, sendo um mínimo de 27 e máximo de 73 anos. Os diagnósticos mais frequentes foram câncer colorretal em 15 pacientes (27\%) e câncer de mama em 12 (22\%). Os protocolos de quimioterapia mais utilizados foram doxorrubicina e ciclofosfamida (AC) e FOLFOX, em 19 (35\%) dos pacientes.

Tabela 1. Características sociodemográfica e clínica da amostra $(n=55)$

\begin{tabular}{|c|c|}
\hline Características & Frequência* \\
\hline \multicolumn{2}{|l|}{ Sociodemográficas } \\
\hline \multicolumn{2}{|l|}{ Sexo* } \\
\hline Feminino & $32(58)$ \\
\hline Idade (em anos) ${ }^{* *}$ & $54,1(12,2)$ \\
\hline Anos de estudo** & $8,5(3,8)$ \\
\hline \multicolumn{2}{|l|}{ Estado civil* } \\
\hline Casado & $24(44)$ \\
\hline Divorciado & $15(27)$ \\
\hline Solteiro & $12(22)$ \\
\hline Viúvo & $4(7)$ \\
\hline \multicolumn{2}{|l|}{ Clínicas* } \\
\hline \multicolumn{2}{|l|}{ Diagnóstico\# } \\
\hline Câncer colorretal & $15(27)$ \\
\hline Câncer de mama & $12(22)$ \\
\hline Câncer de pulmão & $4(7)$ \\
\hline Câncer de esôfago & $4(7)$ \\
\hline Câncer de útero & $3(6)$ \\
\hline Câncer de pâncreas & $3(6)$ \\
\hline Câncer gástrico & $2(4)$ \\
\hline Câncer urogenital & $2(4)$ \\
\hline Linfoma não Hodgkin & $2(4)$ \\
\hline \multicolumn{2}{|l|}{ Protocolo de quimioterapia\#\# } \\
\hline AC - Doxorrubicina e ciclofosfamida & $10(18)$ \\
\hline FOLFOX & $9(16)$ \\
\hline Paclitaxel e carboplatina & $7(13)$ \\
\hline Xelox & $5(9)$ \\
\hline Cisplatina & $4(7)$ \\
\hline Cisplatina e gencitabina & $3(6)$ \\
\hline Etoposido e cisplatina & $3(6)$ \\
\hline Folfirinox & $2(4)$ \\
\hline Cisplatina e fluorouracil & $2(4)$ \\
\hline
\end{tabular}

$\left({ }^{*}\right)$ Variáveis categóricas apresentadas em frequência absoluta e percentual (\%). (**) Variáveis contínuas apresentadas em média e desvio-padrão.

(*) Frequência de $1(2 \%)$ caso de: câncer de próstata, hepático, neuroendócrino, testículo, ovário, melanoma, linfoma Hodgkin e linfoma difuso.

${ }^{(\# \#)}$ Frequência de $1(2 \%)$ dos protocolos de: doxorrubicina, ABVD, paclitaxel, docetaxel, etoposido e carboplatina, cisplatina e vinorelbina, docetaxel e ciclofosfamida, capox, R-CHOP, docetaxel e trastuzumab. 
$\mathrm{Na}$ Tabela 2, consta a comparaçáo entre as duas avaliaçôes realizadas com os instrumentos utilizados. Observou-se que não houve modificação significativa em relação aos sintomas depressivos e de ansiedade, bem como a resiliência e os mecanismos de defesa não variaram significativamente.

Tabela 2. Comparação entre a primeira e a segunda avaliação relacionada aos sintomas depressivos e da ansiedade, resiliência e mecanismos de defesa

\begin{tabular}{|c|c|c|c|}
\hline Variáveis & $\begin{array}{l}\text { Avaliaçãá1 } \\
(n=55)\end{array}$ & $\begin{array}{c}\text { Avaliação } 2 \\
(n=54)\end{array}$ & $\mathbf{p}$ \\
\hline \multicolumn{4}{|l|}{ Sintomas* } \\
\hline Depressivos & $10(5-16)$ & $9(5,7-15)$ & 0,924 \\
\hline Ansiedade & $5(2-12)$ & $5,5(2-10)$ & 0,882 \\
\hline \multicolumn{4}{|c|}{ Escala de Resiliência** } \\
\hline Resiliência & $136,3(28,4)$ & $133(24,9)$ & 0,280 \\
\hline \multicolumn{4}{|c|}{ Mecanismos de defesa** } \\
\hline Madura & $6,1(1,4)$ & $5,8(1,5)$ & 0,059 \\
\hline Neurótica & $5,0(1,3)$ & $4,8(1,2)$ & 0,255 \\
\hline Imatura & $3,8(1,9)$ & $3,8(1,1)$ & 0,705 \\
\hline
\end{tabular}

$\left(^{*}\right)$ Variáveis contínuas apresentadas em mediana e percentis (P25-P75), analisadas com Wilcoxon.

$\left({ }^{* *}\right)$ Variáveis contínuas apresentadas em média e desvio-padrão, analisadas com teste t para amostra dependente.

Por outro lado, verificou-se que houve correlação negativa de moderada a grande entre os níveis de resiliência com os sintomas depressivos $(\mathrm{r}=-0,544)$ e de ansiedade $(\mathrm{r}=-0,402)$ tanto na primeira $(\mathrm{p}<0,001)$ como na segunda avaliação $(\mathrm{p}<0,05)$. Ou seja, quanto maior são os sintomas depressivos e de ansiedade, menor é a resiliência dos pacientes (Tabela 3).

Em relação aos mecanismos de defesa, considerando a segunda avaliação, houve correlaçáo moderada positiva significativa entre os mecanismos maduros e a resiliência. Isto é, quanto maior os mecanismos maduros de humor $(\mathrm{r}=0,535)$, racionalização $(\mathrm{r}=0,482)$ e supressão $(\mathrm{r}=0,317)$, maior foi a resiliência. Não foi encontrada correlação com os mecanismos neuróticos. Já com os mecanismos imaturos, houve correlação moderada negativa com a atuação $(\mathrm{r}=-0,322)$ e de moderada positiva com a dissociação $(r=0,446)$ e cisão $(r=0,307)$.

\section{DISCUSSÃO}

A resiliência refere-se à capacidade de adaptação a adversidades, sendo influenciada por vários fatores, entre eles, presença de depressáo e processos mentais que podem ser usados no enfrentamento de situaçóes difíceis ${ }^{6-8}$. Os resultados do presente estudo evidenciaram a correlação entre a resiliência e os sintomas, sendo que, quanto maior os escores dos sintomas depressivos e ansiosos, menor foi o nível de resiliência.

Neste estudo, foi encontrado resultado semelhante aos achados prévios sobre a presença de sintomas depressivos e de ansiedade, de moderada a grave, sendo frequente em pacientes diagnosticados com câncer ${ }^{13}$. Ainda, os sintomas de ansiedade e depressão influenciam no enfrentamento e na adesão ao tratamento proposto para o câncer ${ }^{14}$.

Confirmando a associaçáa, em um estudo de mulheres com câncer de mama, em estágio inicial, os resultados demonstraram que existe correlação negativa significativa entre depressão e resiliência ${ }^{15}$. Em outro estudo que avaliou qualidade de vida e resiliência, foi encontrado que pacientes com altos escores de resiliência apresentaram melhor qualidade de vida ${ }^{16}$. Os autores enfatizam que os achados podem servir como base para o desenvolvimento de intervençôes psicossociais que fortaleçam a resiliência em pacientes com câncer ${ }^{15,16}$.

Em relação aos mecanismos de defesa, foi encontrado que os mecanismos de humor, racionalização e supressão (maduros), a dissociaçáo e a cisão (imaturos) apresentaram correlação significativa positiva com a resiliência. Já, na atuação (imaturo), a correlação foi inversa. Os mecanismos de defesa podem ser usados no processo de enfrentamento, sendo que os maduros são utilizados para adaptação frente a situaçóes estressantes. Os mecanismos imaturos, por sua vez, podem ser usados para distorção das situaçóes estressoras ${ }^{8,9}$. Ou seja, o paciente consegue expressar seus sentimentos, justificar as situaçóes consideradas como inaceitáveis (por exemplo, o câncer) e reconhecer sua atual situação ao seu tempo, minimizando o impacto por meio do uso de mecanismos de defesa como o humor e a racionalizaçáo. Neste sentido, os mecanismos de defesa mais adaptativos associam-se aos níveis mais elevados de resiliência, facilitando o enfrentamento de situação estressora, como o tratamento com quimioterapia ${ }^{7}$. Segundo uma revisão sistemática ${ }^{8}$ sobre mecanismos de defesa em pacientes oncológicos, a racionalização, a projeção e a negação foram os mecanismos de defesa identificados com frequência nos estudos incluídos. A conclusão dos autores da revisão é de que compreender o funcionamento defensivo de pacientes oncológicos pode auxiliar no aprimoramento da assistência oferecida a eles ${ }^{8}$.

De fato, o tratamento oncológico gera mudanças importantes na vida dos pacientes. Além da própria condiçáo da doença, dos efeitos dos medicamentos ou dos procedimentos, as atividades de vida diária são alteradas. Contudo, em um estudo sobre resiliência em pacientes oncológicos, constatou-se que o tratamento em si contribui para o desenvolvimento da resiliência, promovendo maior utilização dos fatores protetores dos indivíduos ${ }^{3}$. 
Tabela 3. Correlação entre resiliência e sintomas depressivos e de ansiedade e mecanismos de defesa na primeira e segunda avaliação

\begin{tabular}{|c|c|c|c|c|}
\hline \multirow{3}{*}{ Variáveis } & \multicolumn{4}{|c|}{ Resiliência } \\
\hline & \multicolumn{2}{|c|}{ Avaliaçãol ( $n=55)$} & \multicolumn{2}{|c|}{ Avaliação 2 ( $n=54)$} \\
\hline & $\mathbf{r}^{*}$ & $\mathbf{p}$ & $\mathbf{r}^{*}$ & $\mathbf{p}$ \\
\hline \multicolumn{5}{|l|}{ Sintomas } \\
\hline Depressivos & $-0,523$ & $<0,001$ & $-0,544$ & $<0,001$ \\
\hline Ansiedade & $-0,467$ & $<0,001$ & $-0,402$ & 0,003 \\
\hline \multicolumn{5}{|c|}{ Mecanismos de defesa } \\
\hline Maduros & 0,384 & 0,004 & 0,475 & 0,002 \\
\hline Sublimação & 0,149 & 0,278 & 0,113 & 0,417 \\
\hline Humor & 0,368 & 0,006 & 0,535 & $<0,001$ \\
\hline Antecipação & 0,202 & 0,140 & 0,236 & 0,086 \\
\hline Racionalização & 0,322 & 0,017 & 0,482 & $<0,001$ \\
\hline Supressão & 0,197 & 0,150 & 0,317 & 0,019 \\
\hline Neuróticos & 0,068 & 0,620 & 0,052 & 0,710 \\
\hline Anulação & $-0,166$ & 0,227 & $-0,085$ & 0,542 \\
\hline Altruísmo & $-0,073$ & 0,598 & $-0,075$ & 0,589 \\
\hline Idealização & 0,111 & 0,418 & 0,136 & 0,328 \\
\hline Formação reativa & 0,104 & 0,418 & 0,120 & 0,386 \\
\hline Imaturos & $-0,063$ & 0,646 & $-0,027$ & 0,849 \\
\hline Projeção & $-0,235$ & 0,084 & $-0,117$ & 0,398 \\
\hline Isolamento & 0,024 & 0,865 & $-0,003$ & 0,982 \\
\hline Agressão passiva & $-0,363$ & 0,006 & $-0,179$ & 0,194 \\
\hline Atuação & $-0,183$ & 0,180 & $-0,322$ & 0,017 \\
\hline Cisão & 0,100 & 0,469 & 0,307 & 0,024 \\
\hline Fantasia autística & $-0,241$ & 0,076 & $-0,281$ & 0,039 \\
\hline Desvalorização & 0,062 & 0,653 & $-0,178$ & 0,197 \\
\hline Negação & 0,170 & 0,215 & 0,147 & 0,287 \\
\hline Dissociação & 0,333 & 0,013 & 0,446 & 0,001 \\
\hline Deslocamento & $-0,178$ & 0,195 & $-0,057$ & 0,684 \\
\hline Somatização & $-0,234$ & 0,086 & $-0,120$ & 0,349 \\
\hline
\end{tabular}

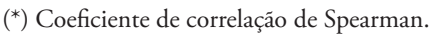

O presente trabalho apresenta limitaçóes que necessitam ser apontadas. Por se tratar de estudo observacional de correlação, não foi possível estabelecer o sentido de causalidade entre as variáveis. Em virtude do curto intervalo de tempo entre as avaliaçôes, não foram detectadas modificaçóes de sintomas de ansiedade e de depressão. No entanto, trata-se de uma pesquisa relevante tendo em vista a escassez de estudos na temática de resiliência na área de quimioterapia ambulatorial.

\section{CONCLUSÃO}

De acordo com as pesquisas, o câncer é uma doença atual e prevalente que, a cada ano, incide na população mundial. Uma das modalidades de tratamento é a quimioterapia, que acarreta efeitos indesejáveis ao indivíduo. Nesse sentido, os pacientes enfrentam mudanças intensas na vida social, profissional e emocional. Tais efeitos adversos do tratamento geram desconforto, assim, o indivíduo necessita buscar meios para enfrentar as modificações e o estresse.

A contribuição deste estudo, que avaliou aspectos emocionais por meio de instrumentos validados, foi a confirmação de que há correlação entre a presença de sintomas depressivos e de ansiedade com a capacidade de enfrentamento. A resiliência mostra-se como crucial para o tratamento e, de acordo com os resultados aqui apresentados, as emoçóes do paciente interferem nos níveis de resiliência. Também foi possível verificar que os mecanismos de defesa utilizados durante o tratamento 
se correlacionaram com a maneira de enfrentamento da situação estressora, no caso, o tratamento quimioterápico.

Enfim, considerando que a resiliência é passível de modificação, é importante que a equipe de multiprofissional atente-se aos aspectos psicossociais do paciente ao longo das sessóes terapêuticas para definir a necessidade de acompanhamento específico para a saúde mental. Dessa forma, torna-se viável compreender e cuidar do paciente de maneira eficiente, integral e qualificada. Estudos de seguimento para avaliar a resiliência e os fatores associados mostram-se necessários para compreensão dos processos mentais de pacientes oncológicos.

\section{CONTRIBUIÇÕES}

Todos os autores contribuíram substancialmente na concepção e/ou planejamento do estudo; na obtenção, análise e/ou interpretação dos dados; na redação e revisão crítica; e aprovaram a versão final a ser publicada.

\section{DECLARAÇÃO DE CONFLITO DE INTERESSES}

Nada a declarar.

\section{FONTES DE FINANCIAMENTO}

Não há.

\section{REFERÊNCIAS}

1. Instituto Nacional de Câncer José Alencar Gomes da Silva. ABC do câncer: abordagens básicas para o controle do câncer. 6. Ed. Rio de Janeiro: INCA; 2020.

2. Ferreira AM, Santos Y, Chagas CB, et al. Processo de enfermagem aplicado a pacientes oncológicos submetidos à quimioterapia e radioterapia ambulatorial. In: Guaragna BFP, Tigre A, Nascimento IM, organizadores. Práticas em oncologia: uma abordagem para enfermeiros e profissionais de saúde. Porto Alegre: Moriá Editora; 2020. p. 29-50.

3. Rodrigues FSS, Polidori MM. Enfrentamento e resiliência de pacientes em tratamento quimioterápico e seus familiares. Rev Bras Cancerol. 2012;58(4):61927. doi: https://doi.org/10.32635/2176-9745. RBC. $2012 \mathrm{v} 58 \mathrm{n} 4.564$

4. Ferreira AS, Bicalho BP, Neves LFG, et al. Prevalência de ansiedade e depressão em pacientes oncológicos e identificação de variáveis predisponentes. Rev Bras Cancerol. 2016;62(4):321-28. doi: https://doi. org/10.32635/2176-9745.RBC.2016v62n4.159

5. Menezes IC, Juruena MF. Diagnóstico de depressóes unipolares e bipolares e seus especificadores. Medicina
(Ribeirão Preto) 2017;50(Supl.1):64-71. doi: https://doi. org/10.11606/issn.2176-7262.v50isupl1.p64-71

6. Oliveira ME. Assistência psicológica ao paciente oncológico. In: Guaragna BFP, Tigre A, Nascimento IM, organizadores. Práticas em oncologia: uma abordagem para enfermeiros e profissionais de saúde. Porto Alegre: Moriá Editora; 2020. p. 281-300.

7. Soratto MT, Silva DM, Zugno PI, et al. Espiritualidade e resiliência em pacientes oncológicos. Saúde e Pesqui. 2016;9(1):53-63. doi: https://doi.org/10.17765/19831870.2016v9n1p53-63

8. Di Giuseppe M, Ciacchini R, Micheloni T, et al. Defense mechanisms in cancer patients: a systematic review. J Psychosom Res. 2018;115:76-96. doi: https://doi. org/10.1016/j.jpsychores.2018.10.016

9. Blaya C, Dornelles M, Blaya R, et al. BrazilianPortuguese version of defensive style questionnaire- 40 for the assessment of defense mechanisms: construct validity study. Psychother Res. 2007;17(3):261-72. doi: https:// doi.org/10.1080/10503300500485581

10. Gorenstein C, Andrade L. Validation of a portuguese version of the Beck Depression Inventory and the StateTrait Anxiety Inventory in Brazilian subjects. Braz J Med Biol Res. 1996;29(4):453-7.

11. Pesce RP, Assis SG, Avanci JQ, et al. Adaptação transcultural, confiabilidade e validade da Escala de Resiliência. Cad Saúde Pública. 2005;21(2):436-8. doi: https://doi.org/10.1590/S0102-311X2005000200010

12. Psychometrica [Internet]. Dettelbach (Germany): Psychometrica; c2016-2022. Lenhard W, Lenhard A. Calculation of effect sizes; 2016. doi: https://doi. org/10.13140/RG.2.2.17823.92329

13. Simão DAS, Aguiar ANA, Souza RS, et al. Qualidade de vida, sintomas depressivos e de ansiedade no início do tratamento quimioterápico no câncer: desafios para o cuidado. Enferm Foco. 2017;8(2):82-6. doi: https://doi. org/10.21675/2357-707X.2017.v8.n2.874

14. Grandizoli MV, Ibiapina ISM, Santos Junior R, et al. Indicadores de esperança, ansiedade e depressão de pacientes em tratamento oncológico. Arq Ciênc Saúde. 2017;24(3):65-70.

15. Ristevska-Dimitrovska G, Stefanovski P, Smichkoska S, et al. Depression and resilience in breast cancer patients. Open Access Maced J Med Sci. 2015;3(4):661-5. doi: https://doi.org/10.3889/oamjms.2015.119

16. Ristevska-Dimitrovska G, Filov I, Rajchanovska D, et al. Resilience and quality of life in breast cancer patients. Open Access Maced J Med Sci. 2015;3(4):727-31. doi: https://doi.org/10.3889/oamjms.2015.128

Recebido em 18/2/2021 Aprovado em 5/5/2021 